\title{
Variations in Spottedness of Photosphere and Chromosphere of the Flare Star EV Lac
}

\author{
R.E. Gershberg, I.V. Ilyin and N.I. Shakhovskaya \\ Crimean Astrophysical Observatory \\ Nauchny, Crimea 334413, USSR
}

\begin{abstract}
Quantitative analysis of the variations of EV Lac as being caused by spottedness leads to the conclusion that the total areas of starspots vary strongly - up to two or threefold - from one season to another but the starspot temperatures remain constant.
\end{abstract}

During 9 nights in September 1986 and 1987 we carried out photometrical and spectroscopical observations of the flare star EV Lac. Excluding flare occurrence time intervals, we obtained data allowing the detection of variations of stellar photosphere and chromosphere. The photometric data are shown in Fig. 1 and the spectroscopic data in Fig. 2. From these plots the following qualitative conclusions are made.

First, in 1986 periodic brightness variations occurred with an amplitude $\Delta V=$ 0 m 15 and in 1987 the amplitude decreased to 0 m 07 .

Second, the phases of periodic brightness variations in these two seasons differ by about half of the period and neither of them coincides with the phase in 198081.

Third, in 1986 the maximum brightness of EV Lac was higher by at least 0.055 than in 1987, and in 1986 the minimum brightness of the star was about 0.025 lower than in 1987. This means that the least spotted hemisphere in 1986 was less spotted than the least spotted one in 1987, and the most spotted hemisphere in 1986 was more spotted than the most spotted one in 1987.

Fourth, in 1986 a clear anti-correlation between $\mathrm{H} \alpha$ emission line intensity and stellar optical brightness was observed; in 1987 such an anti-correlation disappeared. However, the $\mathrm{H} \alpha$ line intensity in 1987 was close to the highest level of 1986.

These four points lead to the conclusion that the decrease of the periodic brightness variation amplitude between 1986 and 1987 is due to more homogeneous distribution of dark spots over the stellar surface with the increase of total area of starspots. In this case an equatorial belt of spots seems much more probable than several high latitude or polar spots.

The quantitative analysis of the data obtained leads to following results. 


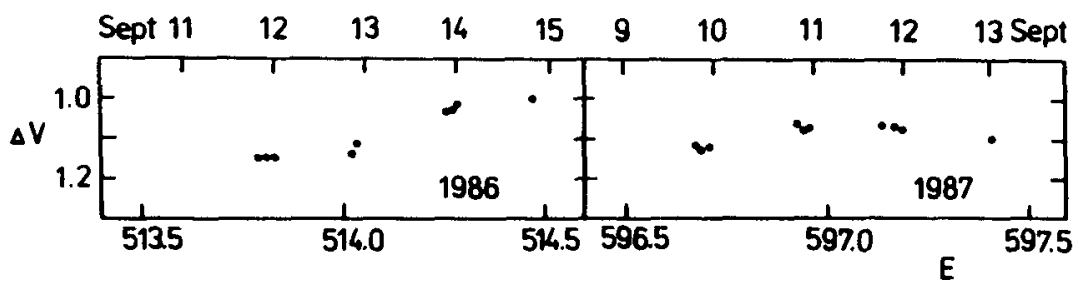

Fig. 1. Light curves of EV Lac: $\Delta V$ is a difference between stellar magnitudes of EV Lac and of the comparison star SAO 52337, $E$ is a period number according to Rojzman's ephemeris (1984).

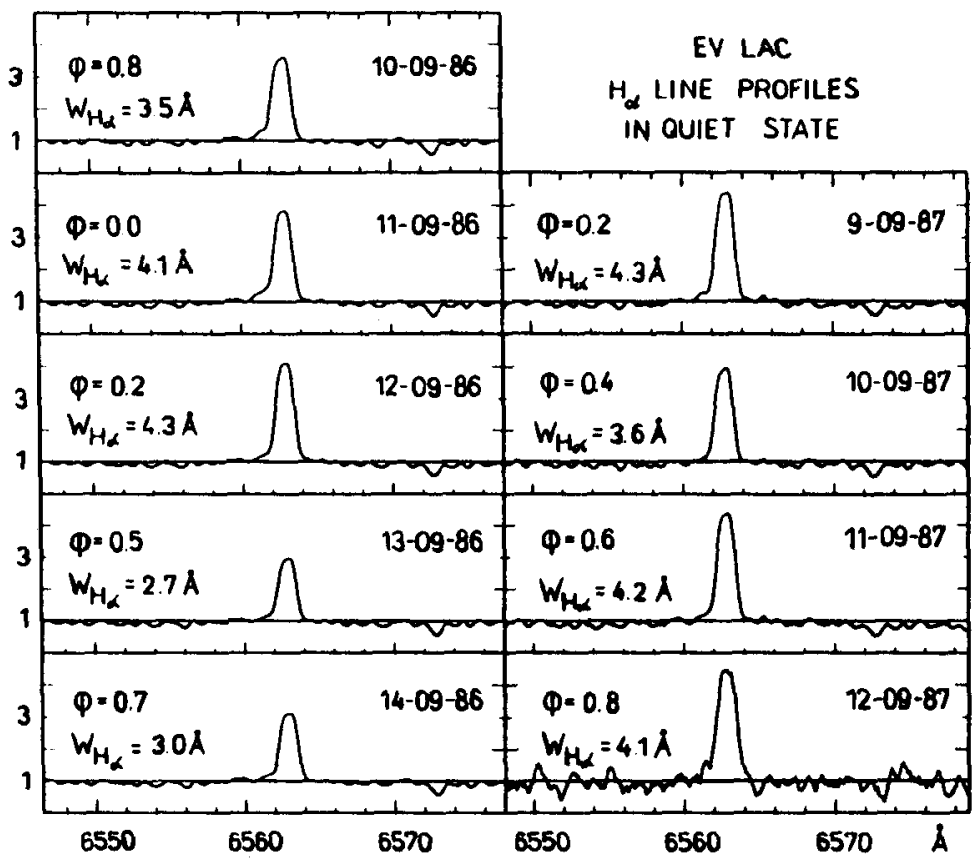

Fig. 2. Nightly averaged $\mathrm{H} \alpha$ emission line profiles in EV Lac spectra during quiet state; $\varphi$ is a phase with reference to brighness minima in Fig. 1.

Brightness variations in different pass bands for the EV Lac starspots show the correlations:

$$
\begin{cases}\Delta U / \Delta V=0.82 \pm 0.12 & \mathrm{r}=0.88 \\ \Delta B / \Delta V=1.06 \pm 0.05 & \mathrm{r}=0.98 \\ \Delta R / \Delta V=0.60 \pm 0.13 & \mathrm{r}=0.76 \\ \Delta I / \Delta V=0.42 \pm 0.03 & \mathrm{r}=0.96\end{cases}
$$

Following Torres and Ferraz Mello (1973) and others, we model the brightness variations from starspots by the relation 


$$
\Delta m_{\lambda}=2.5 \lg \left[1-\alpha\left(1-\beta_{\lambda}\right)\right],
$$

$\alpha$ being a relative spot area and $\beta_{\lambda}=\left(F_{\text {spot }} / F_{\text {photosphere }}\right)_{\lambda}$. The ratio of specific fluxes is usually computed by the Planck formula or by energy distributions from spectra of a set of stars of different spectral classes. We assume too that starspots radiate as cooler stars. However, we take $\beta_{\lambda}$ values only from photometric data. For this purpose we used Pettersen's $(1976,1980)$ data on stellar magnitudes and radii for $\mathrm{K}$ and $\mathrm{M}$ dwarfs and calculated values that may be called radius-unified absolute stellar magnitudes:

$$
m_{\lambda}^{\prime}=m_{\lambda}+\left(M_{V}-m_{V}\right)+5 \lg \left(R_{*} / R_{0}\right) .
$$

Then we found close correlations between variations of these stellar magnitudes along the spectral sequence:

$$
\begin{cases}\Delta U^{\prime} / \Delta V^{\prime}=1.23 \pm 0.04 & \mathrm{r}=0.99 \\ \Delta B^{\prime} / \Delta V^{\prime}=1.15 \pm 0.02 & \mathrm{r}=0.99 \\ \Delta R^{\prime} / \Delta V^{\prime}=0.67 \pm 0.04 & \mathrm{r}=0.97 \\ \Delta I^{\prime} / \Delta V^{\prime}=0.35 \pm 0.04 & \mathrm{r}=0.92 .\end{cases}
$$

According to Poe and Eaton (1985), the $V$ and $I$ bands are the most suitable for starspot investigations. Noting that the last line in (4) leads to $\lg \beta_{I} / \lg \beta_{V}=0.35$, we can obtain from (1) and (2) relations defining $\alpha$ and $\beta_{V}$ :

$$
\begin{aligned}
\beta_{V} & =1-\frac{1-\operatorname{dex}(-0.4 \Delta V)}{1-\operatorname{dex}(-0.168 \Delta V)}\left(1-\beta_{V}^{0.35}\right), \\
\alpha & =\frac{1-\operatorname{dex}(-0.4 \Delta V)}{1-\beta_{V}} .
\end{aligned}
$$

Applying relations (5) to our photometry, we find $\alpha=0.25$ and $\beta_{V}=0.48$ (or $\Delta T=280 \mathrm{~K}$ ) for 1986 and $\alpha=0.13$ and $\beta_{V}=0.51$ for 1987 ; these parameters characterize differences between bright and dark hemispheres of the star in these seasons. Applying relations (5) to the Mavridis and Avgoloupis (1986) data, we find $\alpha=0.39$ and $\beta_{V}=0.42$. These parameters characterize the difference between mean spottedness of the star in 1975 and 1979. Thus, we have found large variations in total starspot areas but practically the starspot temperatures are almost constant in different seasons.

\section{References}

Mavridis, L.N., Avgoloupis, S.: 1986, Astron. Astrophys. 154, 171

Pettersen, B.R.: 1976, Institute Theor. Astrophys. Report N 46, Blindern, Oslo

Pettersen, B.R.: 1980, Astron. Astrophys. 82, 53

Poe, C.H., Eaton, J.A.: 1985, Astrophys. J. 289, 644

Rojzman, G.Sh.: 1984, Pis'ma Astron. Zh. 10, 279

Torres, C.A.O., Ferraz Mello, S.: 1973, Astron. Astrophys. 27, 231 